\title{
Social evolution of shared biofilm matrix components
}

2

3

$4 \quad$ Jung-Shen B.

5

6

7

8

9

10

11

12

13

14

15

16

17

18

19

20

16

8

9

0

${ }^{1}$ Department of Molecular, Cellular and Developmental Biology

${ }^{5}$ Quantitative Biology Institute

Yale University, New Haven, CT 06511, USA.

${ }^{2}$ Department of Mechanical Engineering

University of Minnesota, Minneapolis, MN 55455, USA.

${ }^{3}$ Department of Molecular Biology and Biochemistry

Wesleyan University, Middletown, CT 06459, USA

${ }^{4}$ Department of Biological Sciences

Dartmouth College, Hanover, NH 03755, USA. 


\section{Abstract}

22 Biofilm formation is an important and ubiquitous mode of growth among bacteria. Central to the

23 evolutionary advantage of biofilm formation is cell-cell and cell-surface adhesion achieved by a

24 variety of factors, some of which are diffusible compounds that may operate as classical public

25 goods - factors that are costly to produce but may benefit other cells. An outstanding question is

26 how diffusible matrix production, in general, can be stable over evolutionary timescales. In this

27 work, using Vibrio cholerae as a model, we show that shared diffusible biofilm matrix proteins

28 are indeed susceptible to cheater exploitation, and that the evolutionary stability of producing these

29 matrix components fundamentally depends on biofilm spatial structure, intrinsic sharing

30 mechanisms of these components, and flow conditions in the environment. We further show that

31 exploitation of diffusible adhesion proteins is localized within a well-defined spatial range around

32 cell clusters that produce them. Based on this exploitation range and the spatial distribution of cell

33 clusters, we construct a model of costly diffusible matrix production and relate these length scales

34 to the relatedness coefficient in social evolution theory. Our results show that production of

35 diffusible biofilm matrix components is evolutionarily stable under conditions consistent with

36 natural biofilm habitats and host environments. We expect the mechanisms revealed in this study

37 to be relevant to other secreted factors that operate as cooperative public goods in bacterial 38 communities, and the concept of exploitation range and the associated analysis tools to be 39 generally applicable.

\section{Introduction}


42 Biofilms are an ancient and ubiquitous form of bacterial life in which cells form surface-attached

43 groups embedded in an extracellular matrix ${ }^{1}$. Biofilm-dwelling bacteria gain survival advantages

44 from the matrix including resistance to chemical and biological threats such as antibiotic exposure,

45 host immune system defenses, and invasion and predation by other species ${ }^{2-6}$. Biofilm formation

46 also contributes to the pathogenicity of many infectious bacterial species, and thus makes an

47 outsized impact on human health ${ }^{7-10}$. The evolutionary advantage of biofilm formation is often

48 tied to cell-cell and cell-surface adhesion, through which biofilm-forming species gain strength in

49 numbers, resistance to physical disturbance, and physical association with favorable surfaces ${ }^{11,12}$.

50 Indeed, in the native habitats of many biofilm-formers, nutrient distribution is heterogeneous and

51 often localized around solid substrates ${ }^{13-15}$. Among the components of the extracellular matrix,

52 exopolysaccharides and accessory proteins have been suggested to play dominant roles in biofilm

53 adhesion to both biotic and abiotic surfaces in different species ${ }^{3}$.

In order to function properly, the adhesive molecules associated with biofilm matrix must

55 be secreted outside of cells. This raises an important ecological question: is the production of

56 adhesive molecules exploitable, and if so, what conditions allow for their evolutionary stability?

57 In many contexts, secreted beneficial substances that diffuse away from producing cells can be

58 scavenged by exploitative mutants (often termed cheaters), leading to a public goods dilemma ${ }^{16,17}$

59 in which cooperative behaviors partially or completely break down because producing cells, which

60 pay a metabolic cost, are outcompeted by exploitative mutants ${ }^{17-20}$. The diffusive nature of

61 secreted public goods necessarily leads to a characteristic length scale over which they can be

62 shared, or exploited ${ }^{21-24}$. Previous literature has introduced the idea that secretion of public goods

63 can be stabilized against exploitation if this length scale is smaller than or similar to the scale over

64 which clonemates are clustered together, allowing cooperative cells to preferentially help each 
65 other relative to the population as a whole $\mathrm{e}^{25-29}$. This principle is directly analogous to the classical statement of social evolution theory that cooperation can be favored by natural selection when the

67 benefits of receiving cooperative help, weighted by the relatedness coefficient among givers and 68 receivers of cooperation, exceed the costs of cooperation ${ }^{30}$.

Several studies have explored the competitive dynamics of partially shared matrix 70 components in colonies on agar or pellicles at the air-liquid interface ${ }^{31-35}$, which can serve as 71 excellent experimental platforms; but the continuous film of bacteria formed in these cases does 72 not always correspond to scenarios in nature where isolated cell clusters are separated from each 73 other in space. In many cases, biofilm-forming bacteria form cell clusters due to constraints on 74 movement or mother-daughter cell adhesion, and these clusters may be sparsely or densely 75 distributed in space depending on environmental conditions ${ }^{36}$. The physical size of these clonal 76 clusters and the distance between them, therefore, represent two independent length scales. In 77 principle, these two length scales should be compared with the diffusive length scale of the public goods to determine the physical conditions that stabilize cooperative product secretion against 79 cheating.

The biofilm-producing pathogen and marine microbe Vibrio cholerae offers an excellent model for studies that may provide a close coupling of theory and quantitative empirical testing

82 on the distance-dependent cooperative behavior. $V$. cholerae produces a suite of matrix 83 components - including vibrio polysaccharide (VPS) and the proteins RbmA, RbmC, and Bap1 84 that have been extensively characterized. VPS and RbmA are shared in a restricted fashion within 85 secreting cell lineage groups and thus minimally susceptible to exploitation by non-producing 86 cells ${ }^{4,37-40}$; this pattern has also been observed for major matrix components in Pseudomonas 87 aeruginosa and Bacillus subtilis ${ }^{33,34,41,42}$. On the other hand, RbmC and Bap1, which are 
88 particularly important for adhesion of cell clusters to underlying surfaces, can diffuse far away

89 from producing cells $\mathrm{s}^{37,43}$. Here we establish that these two matrix proteins are exploitable by non-

90 producing cells, creating an associated public goods dilemma. Competition assays between

91 producers and non-producers of $\mathrm{RbmC}$ and Bap1 in static culture and with flow reveal the

92 conditions under which populations of adhesion protein producers can resist invasion by cheating

93 cells. We further show that exploitation of these matrix components takes place within a

94 quantifiable spatial range, which depends critically on rates of diffusion and advection. As a result,

95 sparse distribution of cell groups, and environmental flow that limits the spatial range of shared

96 products, work jointly to limit exploitation of the diffusible matrix. This resolution links well to

97 the ecology of $V$. cholerae biofilms in natural habitats and can be straightforwardly related to

98 fundamental social evolution theory ${ }^{30}$.

101 Results

\section{RbmC and Bap1 are exploitable public goods in $V$. cholerae biofilms}

To study competition between diffusible matrix ( $\mathrm{RbmC}$ and Bap1) producers and potential

104 cheaters in $V$. cholerae biofilms, we used a strain background ( $\left.v p v C^{W 240 R}\right)$ locked in a high level constitutive biofilm-producing background allowed us to perform biofilm culturing experiments more readily while avoiding confounding factors from biofilm gene regulation, but our results can be obtained with a wild-type $V$. cholerae background as well (Fig. S1). We generated an isogenic mutant with double deletions of $r b m C$ and bapl as the cheating strain, because of their functional 
110

111

112

113

114

115

redundancy in conferring surface adhesion ${ }^{37,43,45}$. The producer and cheater each expressed a different fluorescent protein to distinguish them from each other (Fig. S2). We first examined whether there is a fitness cost in producing $\mathrm{RbmC}$ and Bapl by comparing producer and cheater growth rates in monoculture. As shown in Figure 1a, the growth rate of the producer is significantly lower than that of the cheater, demonstrating a substantial cost of producing these matrix components.

To document the degree of retention versus diffusive sharing of $\mathrm{RbmC}$ and Bap1, we performed a quantitative Western assay and found that $83 \%$ of $\mathrm{RbmC}$ and $68 \%$ of Bap1 were retained within producer biofilms, with the remaining protein detected in the surrounding liquid medium (Fig. 1b). Recapitulating prior literature, this result confirms that a substantial fraction of $\mathrm{RbmC}$ and Bap1 molecules can leave the cell clusters that produce them ${ }^{37,43}$. Next, to confirm that the molecules diffusing into the medium can be exploited by the cheaters, we tagged the Cterminus of $\mathrm{RbmC}$ or Bap1 with a $3 \times \mathrm{FLAG}$ epitope and followed their spatial distributions in cocultured biofilms using in situ immunostaining. The staining results show that significant amounts of $\mathrm{RbmC}$ and Bap1 are relocated from producer to cheater biofilm clusters (Fig. 1c and Fig. S3).

To determine whether exogenously acquired Bap1 and $\mathrm{RbmC}$ are functional in adhering cheater cell clusters to the substratum, we grew producer and cheater biofilms separately and in co-culture in 96-well plates. After growing biofilms statically for $16 \mathrm{~h}$, we compared the biofilm biomass remaining on the glass substratum after a washing step that removes any weakly-adherent cell clusters (see Methods) (Fig. 1d). We found that in monocultures, almost all producer cell clusters remained surface-bound after washing, whereas nearly zero biomass was found for cheater biofilms after the disturbance, consistent with the absence of surface adhesion in the cheater strain. In contrast, in the co-culture of producer and cheater, some of the cheater cell groups were able to 
133 resist the strong fluid flow during the washing step. Coupling this observation with the

134 immunostaining results, we inferred that adhesion can be partially restored in cheater biofilms by 135 taking advantage of the adhesion proteins produced by the cohabitating producers. Overall, these 136 results confirm that the shared matrix proteins $\mathrm{RbmC}$ and Bap1 in $V$. cholerae biofilms are 137 cooperative products that draw a significant cost to produce and are susceptible to cheater 138 exploitation.

Exploitation range and community spatial structure jointly determine the outcome of competition between producers and cheaters that non-producing cells could outcompete producing cells in co-culture. To test if this is true, we first competed the producer against cheater in a static environment. The initial biomass of both strains was quantified by the inoculation number densities $\left(\sigma_{0, \mathrm{p}}\right.$ and $\sigma_{0, \mathrm{c}}$ for producer and cheater, 145 respectively; $\sigma_{0} \equiv \sigma_{0, \mathrm{p}}+\sigma_{0, \mathrm{c}}$ is the total inoculation number density). After $16 \mathrm{~h}$ of growth, the total biomass in three-dimensional (3D) space was quantified both before and after washing

147 (Methods; Fig. S4). The competition assay was initiated at different initial frequencies of the 148 producer $f_{0, \mathrm{p}} \equiv \sigma_{0, \mathrm{p}} /\left(\sigma_{0, \mathrm{p}}+\sigma_{0, \mathrm{c}}\right)$ for a range of total inoculation number density $\sigma_{0}$. 149 Representative images and quantitative outcomes of experiments are shown in Fig. 2a-d, where $\Delta f_{1, \mathrm{p}} \equiv f_{1, \mathrm{p}}-f_{0, \mathrm{p}}$ and $\Delta f_{2, \mathrm{p}} \equiv f_{2, \mathrm{p}}-f_{0, \mathrm{p}}$ are the frequency changes of the producer compared to $f_{0, \mathrm{p}}$ before and after washing, respectively. 
155 156

157 158 159 160

161

and the cheater has a substantial advantage in growth rate (Fig. 1a); also, importantly, the benefit of shared adhesion protein production to biofilm cells does not manifest in a disturbance-free environment. The decrease in producer fitness with decreasing $\sigma_{0}$ is due to finite resources in the static environment: At high $\sigma_{0}$, both strains can only undergo a short period of growth before reaching the environment's carrying capacity, limiting the total change in relative abundance of the two strains that can occur. A classic resource-limited competition model reproduced the competition results in a static environment (Fig. S5).

In the competition data after washing, the producer fitness is systematically increased compared to the competition before washing, suggesting that cheater biomass is preferentially removed compared to producer (Fig. 2d). Importantly, this pattern shifted with $\sigma_{0}$ and corresponded to negative frequency-dependent selection for $\mathrm{RbmC}$ and Bap1 production at $\sigma_{0}<$ 0.001: at low initial coverage, the producer is positively selected at low initial frequency. This is evident in Fig. 2e where the competition data are sorted based on inoculation number density $\sigma_{0}$. We expect that at even lower inoculation number densities, the stable point will move to $f_{0, \mathrm{p}}=1$, i.e. the producer will always win regardless of the initial frequency; obtaining sufficient statistics at such low $\sigma_{0}$ is however experimentally difficult due to limits on field-of-view acquisition with sufficient detail to make accurate measurements. We also note that the magnitude of $\Delta f_{2, \mathrm{p}}-\Delta f_{1, \mathrm{p}}$, the fitness difference in producer before and after washing, increases with decreasing $\sigma_{0}$ (Fig. 2e), hinting at the importance of the distance between producer and cheater clusters for the effect of shear disturbance on population dynamics.

To understand the mechanism underlying the sharing dynamics in the competition assay, we analyzed how exploitation of diffusible matrix adhesion proteins takes place in a spatial context. We observed at low $\sigma_{0}$, cheater biomass is distributed predominantly around isolated producer 
biofilms after washing (Fig. 3a). Based on this observation, we hypothesized that there exists a finite spatial range in which cheater cell clusters are protected against fluid disturbance by $\mathrm{RbmC}$ and Bap1 secreted by the producer (Fig. 3b). We define the radius of this range as the exploitation radius $R$, which we extract by calculating the cross-correlation between the producer and cheater biomass distributions in the co-cultures after washing (Fig. 3c). Conceptually, the farther away a cheater cluster is from a producer cluster, the less likely it persists on the substrate after washing - this leads to a decaying likelihood of finding cheater biomass around producers with increasing distance. By assuming an exponentially decaying cross-correlation, we arrived at an exploitation radius $R$ value of $49 \pm 11 \mu \mathrm{m}$ (Figs. 3c and S6; Methods). Note that this value is significantly larger than the physical size of the producer cell cluster, $r_{0}(\approx 9 \mu \mathrm{m})$. This value agrees with the visual inspection of cheater distribution in Fig. 3a. Using this method, we also confirmed the partial redundancy of RbmC and Bap1 in providing adhesion between surfaces and cell clusters, as both single mutants give a similar exploitation radius (Fig. S7).

Next, to cross-check the value of exploitation radius $R$ and to show how this concept can quantitatively explain the competition dynamics, we constructed a spatial model to interpret the dependence of competition outcome on inoculation number density and initial producer frequency (Methods). Briefly, we assume that each producer cell cluster originates from a randomly distributed founder cell, inoculated at a number density $\sigma_{0, \mathrm{p}}$ (Fig. 3d). As such, cheaters within a distance $R$ from any producer cluster remain adherent after washing; otherwise, they are removed. In this setting, the probability $P_{\text {protection }}$ of a cheater cluster falling into the exploitation radius of any producer cluster and being protected from flow disturbance is related to the total exploitable area provided collectively by all of the producers in the system. To calculate the latter, we treat each producer cell cluster as contributing a disk of area $\pi R^{2}$ of protection (Fig. $3 \mathrm{~d}$ ). Note that these 
201

202

203

204

205

206

207

208

209

210

211

212

213

214

215

216

217

218

219

220

221

222

imaginary disks can overlap with each other, because there is no constraint on the distance between inoculated producer cells. The relation between $\sigma_{0, \mathrm{p}}, R$ and $P_{\text {protection }}$ can be derived from probability theory: $P_{\text {protection }}=1-\exp \left(-\sigma_{0, \mathrm{p}} \cdot \pi R^{2}\right)$ (Fig. 3d; Methods). Importantly, $P_{\text {protection }}$ can be easily extracted from the biomass data before and after washing by identifying $P_{\text {protection }}$ as the portion of cheater biomass remaining adherent after washing (Fig. S4). Fitting

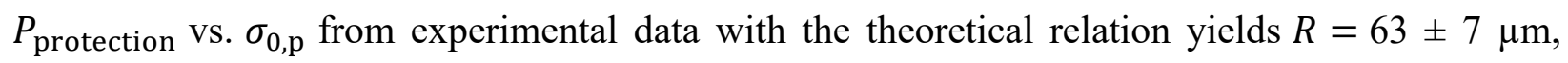
where the error corresponds to $95 \%$ confidence bounds (Fig. 3e). This value is slightly higher than the value extracted above $(49 \pm 11 \mu \mathrm{m})$, but the agreement between the two estimates - one from direct quantification of biomass distribution and the other derived from the spatial exploitation model, is remarkable. The two methods serve as a consistency test for each other and corroborate our hypothesis that exploitation of matrix proteins can take place beyond the physical size of the clonal cluster but only within a finite, well-defined length scale. A two-part model combining the spatial exploitation model with the structureless competition model can qualitatively reproduce all the experimental data in the 96-well plate (Fig. S5).

\section{Flow shifts the competition between producer and cheater by modulating the exploitation}

\section{range of shared adhesion proteins}

Thus far, we have studied the population dynamics of RbmC/Bap1-producing and cheating strains in static liquid environments, but in natural contexts of $V$. cholerae and many other microbes, including on marine detritus (so-called "marine snow") ${ }^{46}$, biofilms grow under continuous fluid flow. To understand how a continuous flow may shift the competition between diffusible matrix producers and cheaters, we conducted competition assays in microfluidic channels with a flow rate of $1 \mu \mathrm{L} / \mathrm{min}$, corresponding to an average flow speed of $694 \mu \mathrm{m} / \mathrm{s}$ (Methods). The flow speed falls 
223 into the range of the measured speed of sinking marine snow particles, the natural context of (non

224 host-associated) V. cholerae and many other marine bacteria ${ }^{47,48}$.

The presence of flow dramatically changed the population dynamics relative to static co-

227 indicating that RbmC/Bap1-producer and cheater strains can coexist under this condition (Fig. 4,

228 a-d). In contrast, at $\sigma_{0}<0.01$, producers outcompeted cheaters regardless of initial population

229 composition. Compared to a static environment, continuous flow generally enhanced the fitness of

230 the RbmC/Bap1-producer against cheating strains (Fig. 2e). For example, in the range

$0.01<\sigma_{0}<0.1$ and $f_{0, \mathrm{p}} \approx 0.5$, an increase in the flow rate from 0 to $1 \mu \mathrm{L} / \mathrm{min}$ increased the

232 fitness of the producer, an effect that saturates beyond $0.5 \mu \mathrm{L} / \mathrm{min}$ (Fig. 4e).

To understand mechanistically how fluid flow shifts the competition between RbmC/Bap1

235 concentration around a producer cluster attached to the surface at different average flow speeds $\bar{U}$

236 ( $\bar{U}=1$ in simulations corresponds to $1 \mu \mathrm{L} / \mathrm{min}$ in experiments). The producer cluster is modeled

237 as a surface-attached hemisphere and secretes matrix molecules at a constant rate from the biofilm-

238 fluid interface (Methods). The simulation results show that, compared to the purely diffusive case,

239 the adhesion protein concentration is drastically reduced around the producer cluster due to

240 advection at a finite flow speed (Fig. 4f). Furthermore, the advective transport results in a comet-

241 tail profile of the protein concentration at the glass surface along the wake of the fluid flow, which

242 we confirmed in the immunostaining experiment performed under the same condition (Fig. 4g).

To explain the difference in fitness of the producers in the static and flow environments,

244 we again applied the concept of exploitation range. Assuming a threshold concentration above 
245 which cheater biofilms receive sufficient adhesion proteins to resist flow, the corresponding 246 effective $R$ around producer cell clusters can be derived based on the simulated concentration

247 profiles of RbmC/Bapl at different flow speeds (Fig. 4f). As $\bar{U}$ increases, the effective $R$ reduces

248 sharply: for example, setting an arbitrary threshold, as shown in Fig. 4h, yields $R=60,22$, and 14

$249 \mu \mathrm{m}$ for $\bar{U}=0,0.1$, and 1 , respectively. The ensuing $P_{\text {protection, }}$ which is dependent on $R$, decreases

250 dramatically with decreasing $R$ as shown in Fig. 4i. Our simulation results thus show that flow

251 shrinks the effective exploitation radius conferred by an individual producer cluster ${ }^{27}$, which in

252 turn reduces $P_{\text {protection }}$ and positively shifts the competitive dynamics toward a regime that

253 increasingly favors RbmC/Bap1-producing cells. The dependence of $P_{\text {protection }}$ on flow therefore

254 qualitatively accounts for the dependence of fitness on flow (Fig. 4e). Combined with the distance

255 between producer cell clusters driven by sparse single cells colonizing the surface, flow limits the

256 spatial range of sharable matrix components and averts exploitation of sharable matrix adhesion

257 proteins.

\section{Discussion}

259 Cell-cell attachment and cell-surface adhesion are often critical to the evolutionary advantage of 260 biofilm formation. The extracellular matrix plays an important role in controlling biofilm 261 organization and adhesion ${ }^{37-39,45,49-54}$, and understanding the evolutionary dynamics of its 262 production remains an active area of work in this research domain. The biofilm matrix often 263 contains components that vary in their diffusion properties and potential for exploitation by cells 264 that do not produce them. In this study, we explore the population dynamics of diffusible matrix 265 protein production in $V$. cholerae biofilms. We carried out competition assays under static and 266 flow conditions, both of which are frequently encountered by $V$. cholerae in native habitats and in

267 hosts $^{47,48,55}$. We found that the matrix components RbmC and Bap1, as diffusible public goods, 
can be exploited and confer resistance to physical disturbance to clusters of non-producing cells within a finite spatial range around producer cell clusters. This exploitation range depends on the diffusion-advection condition of the environment, where a continuous flow effectively shrinks the exploitation range and consequently suppresses cheater exploitation. In their marine and fresh water reservoirs, $V$. cholerae cells often attach to floating food particles and experience shear flows 47,48. During infection, $V$. cholerae biofilms are primarily localized on the tips of intestinal villi and therefore experience peristaltic flow in the gut ${ }^{55}$. The flow speed and the associated shear stress used in this study fall within the range measured in these environments ${ }^{56}$. The population densities of $V$. cholerae cells in natural ecosystems have been measured to range from $10^{2}$ to $10^{5}$ cells per liter ${ }^{57}$, corresponding to an average distance between cells on the order of millimeters in 3D space. Thus, our results suggest that the combination of flow and low cell density drive the competition dynamics in favor of $\mathrm{RbmC}$ and Bap1 producers and lead to the evolutionary stability of these diffusible matrix proteins in $V$. cholerae.

It is interesting to contrast our results with those for the other major matrix protein in $V$. cholerae biofilms, RbmA, which (together with VPS) is responsible for high density cell-cell packing within biofilm cell clusters. RbmA is secreted and shared in a limited fashion within cell lineage groups producing $\mathrm{it}^{4}$, conferring protection from exploitation with little dependence on the distance between clusters of producing versus non-producing cells ${ }^{4}$. We envision that the different sharing dynamics of $\mathrm{RbmA}, \mathrm{RbmC}$, and Bap1 are constrained by their function as components of the biofilm matrix. RbmA, which holds mother-daughter cell lineages together, must be sequestered and stay in close proximity to producing cells to perform this function. RbmC and Bap1, on the other hand, must travel a distance away from producing cells to confer adhesion between cell groups and underlying surfaces, making them inherently sharable and exploitable; 
291 the exact biochemical and biophysical mechanisms underlying this difference await future

292 research. As a consequence, different matrix components - even those produced by cells of one

293 species within the same biofilm - can have different environmental constraints and population

294 structures as conditions for their evolutionary stability.

Our results have a straight-forward correspondence with social evolution theory.

296

297

298

299

300

301

302

303

304

305

306 307 sufficient to stabilize cooperation against cheating ${ }^{25-29}$. Here, however, the spatial range of 308 Hamilton's rule provides the canonical condition under which cooperation is favored by selection: the fitness benefit of receiving cooperative help, weighted by the relatedness coefficient that quantifies the correlation between recipient genotype and cooperative actor genotype ${ }^{58-60}$, must exceed the cost of the cooperative behavior ${ }^{18}$. Putting this principle into the context of our experiments, the cost of $\mathrm{RbmC}$ and Bap1 production is set by their regulation (here, nearly constitutive) and nutrient supply conditions, and the benefit is set by the extent to which shear stress is applied to biofilms (Fig. S8). The relatedness coefficient - taking note again that this effectively measures the extent to which $\mathrm{RbmC/Bap1-producing} \mathrm{cells} \mathrm{benefit} \mathrm{each} \mathrm{other} \mathrm{relative}$ to the total population composition ${ }^{58,60-63}$ - is controlled by the range over which $\mathrm{RbmC}$ and Bap1 diffuse and the spatial distribution of producer and cheater cell clusters ${ }^{26,28,29,58,63,64}$. In many reported cases, the clustering of clonal cell lineages within mixed biofilm communities can be $\mathrm{RbmC/Bap1}$ sharing is larger than the size of clonal cell clusters due to the leakage of these proteins from groups of producing cells, and the associated public goods dilemma cannot be resolved by clonal group clustering alone. Instead, the solution is rooted in the combination of biophysical mechanisms of protein retention and diffusion, community spatial structure, and environmental perturbations, all captured in the spatial exploitation model (Fig. 3). 
The key parameter in our model, the exploitation radius $R$, can additionally depend on many physiological factors including the production rate of the matrix, duration of biofilm growth, aggregation of adhesion proteins, uptake of adhesion protein by cheater matrix, and biofilm 316 dispersal ${ }^{65}$, among other factors. Competition for binding to the exopolysaccharides and for 317 surface adsorption from other biomacromolecules in the environment may also reduce $R^{66}$. In 318 addition, although we have assumed $R$ to be constant in our spatial model of $P_{\text {protection }}, R$ does 319 appear to vary and drop to a much smaller value when the inoculation number density goes above $32010^{-1}$ cells $/ 100 \mu \mathrm{m}^{2}$ (Fig. S9). This can be understood as follows: At a high total inoculation number 321 density, the size of each producer cluster is smaller due to nutrient limitation and the concentration 322 of secreted proteins around each producer cluster is consequently reduced. Additionally, the 323 elevated incorporation of adhesion proteins into the surrounding cheater clusters further constrains 324 the exploitation range. In the limit of a confluent biofilm layer, the system reduces to the well325 studied case of a densely packed bacterial colony ${ }^{21,23}$. To account for the variation of $R$ and

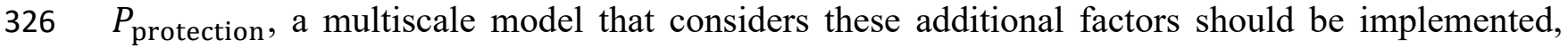
327 which we leave for future studies. Nevertheless, our simplified spatial model already provides a 328 good quantitative description of the underlying dynamics of cooperative matrix protein production, especially in the low seeding density regime most relevant to the natural conditions of $V$. cholerae.

330 While the concept of exploitation range has been discussed in relation to public good production 331 in bacterial communities ${ }^{26-29,67-70}$, direct quantitation of the exploitation radius and a close 332 comparison between model and experiment, as we achieved here, represent important steps 333 forward. Our study thus provides both a conceptual guideline and technical tool set for future 334 studies on how to quantify public goods sharing in relation to population structure in the biofilm 335 context. 
Finally, previous work has shown that in the cases of extracellular enzymes ${ }^{27,71,72}$,

337 siderophores ${ }^{23,73}$, and autoinducers ${ }^{74-76}$ as diffusive public goods, mostly studied in continuous

338 films, clonal segregation and efficient consumption of public goods (or their enzymatic products

339 and complexes) through high local cell density and a high uptake rate can minimize the potential

340 for exploitation in the context of dense bacterial communities ${ }^{21,26,27}$. In the context of spatially

341 discrete clonal clusters, we expect the evolutionary stability of these public goods to be determined

342 by the interplay between the exploitation range and the spatial structure as shown here.

344 Acknowledgments

345 We thank Andrew Sharo, Sara Siwiecki, and Carissa Chan for their help in the initial experiments.

346 We thank Xin Huang, Japinder Nijjer and Qiuting Zhang for helpful discussions. Alexandre Persat,

347 Kevin Foster, Benjamin Wucher, and James Winans provided helpful feedback on the manuscript.

348 S.M. thanks Paul Fischer, Som Dutta, and Nek5000 user group for helpful discussions regarding

349 numerical computations. Numerical computations were performed using the resources of the

350 Minnesota Supercomputing Institute at the University of Minnesota. J.Y. holds a Career Award at

351 the Scientific Interface from the Burroughs Wellcome Fund. C.D.N. is supported by the Simons

352 Foundation award number 826672. J.T. holds a Career Award at the Scientific Interface from the

353 Burroughs Wellcome Fund. J.-S.B.T. is a Damon Runyon Fellow supported by the Damon Runyon

354 Cancer Research Foundation (DRG-2446-21). Research reported in this publication was supported 355 by the National Institute of General Medical Sciences of the National Institutes of Health under 356 Award Number DP2GM146253 (awarded to J.Y.). 


\section{Author contributions}

359 C.D.N. and J.Y. conceived the project. J.-S.B.T. and T.N. performed the experiments. J.Y. 360 performed strain cloning. S.M and J.T. performed fluid dynamics modeling. J.-S.B.T. performed 361 ecological modeling. J.-S.B.T., S.M., J.T., C.D.N. and J.Y. analyzed the data. All authors 362 contributed to the writing of the paper.

364 Corresponding author

365 Correspondence and requests for materials should be addressed to C.D.N.

366 (carey.d.nadell@dartmouth.edu) or J.Y. (jing.yan@yale.edu).

Competition interests

369 The authors declare no competing interests. 
Methods

372 Strains and media

373 All $V$. cholerae strains used in this study are derivatives of the wild-type Vibrio cholerae O1 biovar El Tor 374 strain C6706 and listed in Table S1. All strains harbor a missense mutation in the $v p v C$ gene $\left(v p v C^{\mathrm{W} 240 \mathrm{R}}\right)$ 375 that elevates intracellular c-di-GMP level and are robust biofilm formers ${ }^{44}$. This allows us to focus on the

376

377

378

379

380

381

382 ecological consequences of adhesion protein production in $V$. cholerae biofilms rather than behaviors involving gene regulation. Additional mutations were genetically engineered using the natural transformation method or through the suicide vector pKAS32 $2^{77,78}$. All strains were grown overnight in lysogeny broth (LB) at $37^{\circ} \mathrm{C}$ with shaking. Competition assays were performed in $\mathrm{M} 9$ medium and supplemented with $2 \mathrm{mM} \mathrm{MgSO}_{4}, 100 \mu \mathrm{M} \mathrm{CaCl}_{2}, 0.5 \%$ glucose, and $0.1 \mathrm{mg} / \mathrm{mL}$ of bovine serum albumin (BSA). BSA is required for blocking non-specific surface adsorption in the immunostaining experiments; to be consistent, we include BSA in all competition assays as well.

\section{Protein quantification}

$V$. cholerae strains encoding Bap1-3 $\times$ FLAG or RbmC-3 $\times$ FLAG were grown in culture tubes containing 3 $\mathrm{mL}$ LB and sterile glass beads overnight at $30^{\circ} \mathrm{C}$ with shaking. The next day, cultures were vortexed to break up pellicles and cell clusters and the $\mathrm{OD}_{600}$ was measured. $1 \mathrm{~mL}$ of cell suspensions were transferred to sterile $1.5 \mathrm{~mL}$ microcentrifuge tube and spun at $18,000 \mathrm{~g}$ for $3 \mathrm{~min} .500 \mu \mathrm{L}$ of the cell supernatant were transferred to a fresh $1.5 \mathrm{~mL}$ microcentrifuge tube and the rest was discarded from the pellet. The cell pellets were lysed for $30 \mathrm{~min}$ in $100 \mu \mathrm{L}$ lysis solution [1× Bugbuster (EMD Millipore 70921), lysozyme $(0.1 \mathrm{mg} / \mathrm{mL})$, and benzonase ${ }^{\mathrm{TM}}$ nuclease (Sigma E1014)] and then brought to a final volume of $1 \mathrm{~mL}$ with $1 \times$ PBS. $30 \mu \mathrm{L}$ of each cell suspension was combined with $10 \mu \mathrm{L}$ of $4 \times$ SDS PAGE sample buffer (40\% Glycerol, $240 \mathrm{mM}$ Tris pH6.8, 8\% SDS, 0.04\% Bromophenol Blue, 5\% $\beta$-mercaptoethanol) and boiled for 10 minutes at $95^{\circ} \mathrm{C}$. Samples were run on a 4-15\% Mini-PROTEAN TGX gel (BioRad 4568086) in $1 \times$ SDS PAGE running buffer ( $25 \mathrm{mM}$ Tris, $192 \mathrm{mM}$ Glycine, $1 \% \mathrm{SDS}, \mathrm{pH} 8.3$ ) at $120 \mathrm{~V}$ for 70 minutes at $4^{\circ} \mathrm{C}$. The proteins were transferred to a PVDF membrane (BioRad 1620174) in $1 \times$ transfer buffer $(25 \mathrm{mM}$ Tris, $192 \mathrm{mM}$ Glycine, $10 \%$ methanol, $\mathrm{pH} 8.3$ ) at $100 \mathrm{~V}$ for 1 hour at $4^{\circ} \mathrm{C}$. Membranes were incubated in $5 \%$ milk in TBST overnight at $4^{\circ} \mathrm{C}$ then at room temperature for $1 \mathrm{hr}$. Following incubation, membranes were washed $3 \times 10$ minutes in $1 \times$ TBST (American Bio AB14330-01000). The membranes were blotted using $\alpha$-DYKDDDDK at $0.1 \mu \mathrm{g} / \mathrm{mL}$ (Biolegend 637311) in $1 \times$ TBST with $3 \%$ BSA for $1 \mathrm{hr}$ at room temperature and washed $3 \times 10$ minutes with $1 \times$ TBST. Blots were developed using the Super Signal PLUS Pico West Chemiluminescent Substrate (Thermofisher 34580) for 5 min and pictures taken using a BioRad Chemidoc-MP. Analyses of sample signal were performed in ImageJ. The total signal for whole cell lysate (Pellet) and supernatant (Sup.) were measured by densitometry and each fraction was calculated against the total signal.

\section{Static competition assay with end-point flow disturbance}

$V$. cholerae strains carrying different fluorescent proteins were streaked onto an LB agar plate and grown overnight at $37^{\circ} \mathrm{C}$. Single colonies with a rugose morphology were picked and grown overnight at $37^{\circ} \mathrm{C}$ with glass beads. The LB culture was then vortexed vigorously before back-diluting 30-fold in M9 medium and grown for 3-4 $\mathrm{h}$ with shaking and with glass beads. After another vortexing, the inoculant was washed with fresh M9 medium without glucose 3 times. The inoculant was then bead bashed using a Digital Disrupter Genie with small glass beads (Acid-washed, 425-500 $\mu \mathrm{m}$, Sigma). This procedure ensures that large cell clusters formed in culture were broken apart to allow more accurate measurement of $\mathrm{OD}_{600}$. The inoculant of the two competing strains was then mixed at different ratios and $\mathrm{OD}_{600}$, and $100 \mu \mathrm{L}$ of the 
mixture was added to wells of 96-plates with a \#1.5 coverslip bottom (MatTek). The cells were allowed $1 \mathrm{~h}$ to attach, after which the wells were washed twice with fresh M9 medium. The wells were subsequently filled with $100 \mu \mathrm{L} \mathrm{M} 9$ growth medium with glucose and $0.1 \mathrm{mg} / \mathrm{mL}$ of BSA and imaged by a confocal fluorescence microscope (see below) to quantify the inoculation number density for both strains. Inoculation number densities, instead of $\mathrm{OD}_{600}$, were used to quantify $f_{0, \mathrm{p}}$ due to the asymmetry in inoculation efficiency between producer and cheater (Fig. S10). The cultures were then grown for 16-18 h at $30^{\circ} \mathrm{C}$ before biomass quantification. After growth, one set of biomass quantification for both strains was done by fluorescence confocal microscopy before a flow disturbance to the culture was introduced by washing the well vigorously twice with fresh medium, after which another set of biomass quantification was done. Immunostaining of adhesion proteins was done using producer strains expressing C-terminal $3 \times$ FLAG-tagged $\mathrm{RbmC}$ or Bap1 co-cultured with the cheater under the same growth conditions for competition assays, except that the growth medium contained Cy3-conjugated anti-FLAG antibodies (2 $\mu \mathrm{g} / \mathrm{mL}$; Sigma-Aldrich).

\section{Competition assay under flow}

The inoculants of the competition strains were prepared in the same way as described in the static competition assay. The inoculants were then introduced into microfluidic channels (channel dimensions: 1 $\mathrm{cm}$ in length, $400 \mu \mathrm{m}$ in width and $60 \mu \mathrm{m}$ in height) through the outlet without inoculating the inlet. This ensures that no biofilms were grown in the zero-flow zone directly beneath the inlet. The cells were allowed $2 \mathrm{~h}$ to attach, after which sterile inlet and outlet polytetrafluoroethylene tubing were connected to the microfluidic chamber and the M9 growth medium was flown through the channel at flow rates controlled by a syringe pump. The flow rates used in this study range from 0.1 to $1 \mu \mathrm{L} / \mathrm{min}$, corresponding to average flow speeds from 69.4 to $694 \mu \mathrm{m} / \mathrm{s}$ and shear rates from 7 to $70 \mathrm{~s}^{-1}$. The microfluidic channels were transferred to a confocal fluorescence microscope and imaged to quantify the inoculation number density for both strains. The cultures under flow were then grown for $16-18 \mathrm{~h}$ at $30^{\circ} \mathrm{C}$ before biomass quantification. After growth, one set of biomass quantification for both strains were done by fluorescence microscopy.

\section{Microscopy, biomass quantification, and growth rate measurement}

Fluorescence confocal microscopy was conducted using a Yokogawa W1 confocal scanner unit connected to a Nikon Ti2-E inverted microscope with a Perfect Focus System. Cells constitutively expressing SCFP3A, mNeonGreen, and mScarlet-I cytosolically were excited by lasers at $445 \mathrm{~nm}, 488 \mathrm{~nm}$, and $561 \mathrm{~nm}$, respectively, and the fluorescent signal was recorded by a sCMOS camera (Photometrics Prime BSI) after the corresponding filters. Confocal images were taken using a $60 \times$ water immersion objective $(\mathrm{NA}=1.2)$ at $2 \times 2$ pixel binning and the resulting pixel size was $217 \mathrm{~nm}$. All images presented are raw images rendered with Nikon NIS-Elements. Producers are pseudo-colored red, cheaters are pseudo-colored yellow, and the immunosignals are pseudo-colored cyan throughout the text unless indicated otherwise.

To quantify the inoculation number density before growth, an image at the surface was taken for each wavelength at the center of each well or microfluidic channel. To quantify the biomass after growth, a 3D tiled image with a $3 \mu \mathrm{m}$ step size in $z$ was taken at 3-5 evenly distributed positions within same the area for each inoculation number density. The total height of the 3D image was chosen to include all biomass of the surface-attached biofilm and is $60-72 \mu \mathrm{m}$ in the static competition assay and $60 \mu \mathrm{m}$ for the competition assay under flow.

For accurate segmentation and quantification of the biomass, we used a modified Bernsen's local thresholding method which is less sensitive to depth-dependent intensity variations and contrast reduction (due to pinhole crosstalk and scattering) in the 3D confocal image. Briefly, each 2D image slice first went 
through a constant background subtraction and denoising, and the image contrast was enhanced by subtracting the image by its Gaussian blur. To determine whether a pixel belongs to cells or background, if the contrast in the local neighborhood around a given pixel is higher than the contrast threshold (50 in all data set), the pixel is set to belong to cells if its value is higher than 1.5 times the median value of the neighborhood; otherwise a pixel is set to belong to cells if its value is higher than an intensity threshold determined based on the brightness of the image. Examples of segmented images are shown in Fig. S4. The total area of cells in each 2D image was converted to a number density using the average cell sizes, measured separately at low cell densities for different fluorescence channels. The number densities in each $2 \mathrm{D}$ slice were then added to obtain the total 3D biomass.

Growth rates of producer and cheater biofilms were measured by time-course imaging of biofilm growing in 96-well plate. Confocal images were taken every $2 \mathrm{~h}$ for $40 \mathrm{~h}$ and biomass was quantified as described above at each time point. The effect of confocal imaging was confirmed to be nonsignificant to biofilm growth under the conditions used in the experiment. We extracted growth rates as slopes of fitting lines to the growth curves in semi-log plots in their exponential phase. Carrying capacities used in the competition model were measured as the maximum biomass in the stationary phase.

\section{Quantification of exploitation range and protection probability}

Cross-correlations between producer and cheater biomass distributions were used to quantify the exploitation radius $R$. We model the cheater biomass distribution as being exponentially decaying around a producer cell cluster of radius $r_{0}$ with the decaying constant equal to $R-r_{0}$; this yields an exploitation radius $R$. We found that the cross-correlation between the producer and cheater images gives adequate information to extract information on $r_{0}$ and $R$ (Fig. S6). To obtain cross-correlations from experimental images, inoculant mixtures of producer strain with $\mathrm{OD}_{600}=0.001$ and cheater strain with $\mathrm{OD}_{600}=0.1$ were used for static competition assay with isolated producer clusters. Maximum intensity projections of confocal images for both strains after end-point flow disturbance were thresholded and used for crosscorrelation calculations. The field of view of each image is $\sim 1.8 \mathrm{~mm} \times 1.8 \mathrm{~mm}$. The cross-correlation between producer and cheater was calculated as

$$
X(i, j)=\sum_{m, n} I_{\mathrm{p}}(m, n) \cdot I_{\mathrm{c}}(m-i, n-j),
$$

where $I_{\mathrm{p}}$ and $I_{\mathrm{c}}$ are the binary images for producer and cheater, and $m$ and $n$ are the row and column indices of the image. $X(i, j)$ was then radially averaged and converted to real spatial units. The error in Fig. $3 \mathrm{C}$ was calculated as the standard error from the $\mathrm{N}=4$ replicates. For a negative control of the correlated biomass distribution, we compared the normalized cross-correlation between producer and cheater biofilms with that between producer and producer biofilms (Fig. S6). The normalized cross-correlation is defined as

$$
X_{\text {norm }}(i, j)=\frac{\sum_{m, n}\left[I_{\mathrm{p}}(m, n)-\overline{I_{\mathrm{p}}}\right] \cdot\left[I_{\mathrm{c}}(m-i, n-j)-\overline{I_{\mathrm{c}}}\right]}{\left\{\sum_{m, n}\left[I_{\mathrm{p}}(m, n)-\overline{I_{\mathrm{p}}}\right]^{2} \sum_{m, n}\left[I_{\mathrm{c}}(m-i, n-j)-\bar{I}_{\mathrm{c}}\right]^{2}\right\}^{1 / 2}}
$$

Where $\bar{I}_{\mathrm{p}}$ and $\bar{I}_{\mathrm{c}}$ are the mean values of $I_{\mathrm{p}}$ and $I_{\mathrm{c}}$, respectively.

\section{Spatial exploitation model based on randomly distributed disks}

Assuming each producer cluster contributes a circular disk of protection with area $\pi R^{2}$, the overall protection probability conferred by producer biofilms to cheater biofilms in an area of interest can be modeled by the coverage problem of randomly distributed circular disks ${ }^{79}$ : let there be $n$ interpenetrable 
disks with area $a$ distributed randomly in an area of interest $A$, the uncovered probability of $A$ has a mean value $\left(1-\frac{a}{A}\right)^{n}$. In the limit of large $A$ and $\frac{n}{A} \rightarrow \lambda$, the uncovered probability approaches $\exp (-\lambda a)$. Therefore, for producer clusters with number density $\sigma_{0, \mathrm{p}}$ and exploitation radius $R$, the protection probability reads $P_{\text {protection }}=1-\exp \left(-\sigma_{0, \mathrm{p}} \cdot \pi R^{2}\right)$.

\section{Flow and adhesion protein simulation in a microfluidic environment}

To gain insight into the effect of different fluid flow speeds on the transport of molecules released by the producer, we solve the governing equations of the fluid flow and a coupled scalar transport equation to model the molecular distribution around a producer. We assume the producer cluster is shaped like a solid hemisphere adhered to the bottom surface of a rectangular channel and subjected to fluid flow, as shown in Fig. S11. The fluid flow is governed by the Navier-Stokes and continuity equations, and the molecular transport is described by the coupled advection-diffusion equation, which are respectively given by:

$$
\operatorname{Re}\left(\frac{\partial \vec{u}}{\partial t}+\vec{u} \cdot \vec{\nabla} \vec{u}\right)=-\vec{\nabla} p+\nabla^{2} \vec{u}
$$

$$
\frac{\partial c}{\partial t}+\vec{u} \cdot \vec{\nabla} c=\frac{1}{\mathrm{Pe}} \nabla^{2} c
$$

In these equations, $\vec{u}(x, y, z, t)=(u, v, w)$ is the fluid velocity vector, $p(x, y, z, t)$ is the pressure, and $c(x, y, z, t)$ is the concentration of molecules released by the producer. Eqs. (1-3) are nondimensionalized using a length scale $d=20 \mu \mathrm{m}$, which is the average diameter of a producer cluster, as found in experiments. The time scale used is the advective time scale $\frac{d}{\overline{U^{*}}}$ where $\overline{U^{*}}=694 \mu \mathrm{m} / \mathrm{s}$ is the dimensional average inflow velocity. The concentration is normalized using an arbitrary reference concentration of $c_{0}=$ 1 adhesion molecule $/ \mu \mathrm{m}^{3}$.

There are two important nondimensional parameters that describe the equations. The Reynolds number $\operatorname{Re}=\frac{\overline{U^{*}} d}{v}$ is defined as the ratio of the inertial to the viscous forces, where $v=1 \times 10^{-6} \mathrm{~m}^{2} / \mathrm{s}$ is the kinematic viscosity of water. Our experiments are typically in the range $0.01<\operatorname{Re}<0.1$, meaning viscous effects dominate the dynamics. At these Re, the left-hand side of Eq. (1) can be neglected, reducing to the Stokes equation. The Péclet number, $\mathrm{Pe}=\frac{\overline{U^{*}} d}{D}$ is defined as the ratio of the diffusive time scale to the advective time scale; $D=80 \mu \mathrm{m}^{2} / \mathrm{s}$ is the diffusion coefficient of the adhesion molecules based on the Stokes-Einstein equation and their estimated size. Pe $>1$ suggests fluid advection has a larger role in transporting the molecules than diffusive transport. We vary the Péclet number in the range $0 \leq \mathrm{Pe} \leq$ 173.5 , in agreement with variable experimental conditions of $0<\overline{U^{*}}<694 \mu \mathrm{m} / \mathrm{s}$.

We simulate fluid flow through a channel of dimension $L_{x} \times L_{y} \times L_{z}$ as shown in Fig. S11. The solid hemisphere modeling the producer cluster is positioned at $\left(x=\frac{L_{x}}{2}, y=\frac{L_{y}}{2}, z=0\right)$. In the experiments, the aspect ratio of the channel is $\Gamma_{x} \times \Gamma_{y} \times \Gamma_{z}=500 \times 20 \times 3$, where $\left\{\Gamma_{i}=\frac{L_{i}}{d} \mid i \in x, y, z\right\}$. This large aspect ratio makes simulations computationally expensive, so we instead use a channel of dimensions $\Gamma_{x} \times \Gamma_{y} \times \Gamma_{z}=20 \times 6 \times 3$ in the simulation, which preserves the important cross-stream dimension along the z-axis. 
A no-slip boundary condition is imposed for the top and bottom surfaces of the domain $(\vec{u}=(0,0,0)$ at $z=$ $\left.\left\{0, L_{z}\right\}\right)$. A Dirichlet boundary condition is applied at the inlet $(\vec{u}=(\bar{U}, 0,0)$ at $x=0)$, where $\bar{U}$ is the average nondimensional inflow velocity into the channel scaled by $\bar{U}^{*}$ and $\bar{U}=1$ corresponds to an average velocity of $694 \mu \mathrm{m} / \mathrm{s}$. An open/outflow boundary condition is applied to the outlet at $x=L_{x}$. Along the material walls at $y=\left\{0, L_{y}\right\}$, we impose a free-slip or no-penetration boundary condition. The boundary condition for the concentration is no-flux on all material walls, except for the surface of the solid hemisphere, for which we use a time-varying Dirichlet boundary condition of the form,

$$
\frac{d c}{d t}=\frac{d}{c_{0} \overline{U^{*}}} k_{r}(1-f)
$$

Here, $k_{r}=0.015$ molecules $/ \mu \mathrm{m}^{3} / \mathrm{s}$ is the total production rate of the molecules from the surface of the producer cluster ${ }^{43}$ and $f$ quantifies the fraction of molecules retained in the producer cluster based on experimentally measured values. Equation (4) suggests that there is a constant rate of production of molecules at the surface of the hemisphere.

We numerically solve the governing equations (1-4) using the spectral element fluid solver NEK5000 $0^{80}$. NEK5000 has been used to study a broad range of fluid flow problems ${ }^{81}$, including complicated chaotic fluid flows and reaction-advection-diffusion systems ${ }^{82-84}$. Our approach uses a semi-implicit operatorsplitting scheme which is $2^{\text {nd }}$ order accurate in time and exponentially convergent in space. Our domain consists of 392 hexahedral spectral elements, and we have used a $17^{\text {th }}$ order Lagrangian interpolant polynomial for spatial discretization, which we found is sufficient to resolve both the fluid flow and the intricate features of the molecular distribution around the producer.

To resolve the small Re of the flow, we evolve Eqs. (1-2) using an extremely small time step of $\Delta t=$ $1 \times 10^{-8}$. We simulate the flow field for $1.4 \times 10^{6}$ steps and ensure that the flow field reaches a steady state. We then stop the evolution of the fluid flow and use the steady velocity profile to solve the advectiondiffusion equation (3). Since we do not evolve the flow field, we use larger time steps (typically $\Delta t=$ $1 \times 10^{-3}$ ) to evolve the concentration field for a total of $3 \mathrm{~min}$ (Fig. S12).

\section{Statistics}

Errors correspond to SEs from measurements taken from distinct samples unless mentioned otherwise. Standard $t$-tests were used to compare treatment groups and are indicated in each figure caption. Tests were always two-tailed and unpaired as demanded by the details of the experimental design. All statistical analyses were performed using GraphPad Prism software. 


\section{Figures}

d $\times 222 \times 60 \mu \mathrm{m}$.
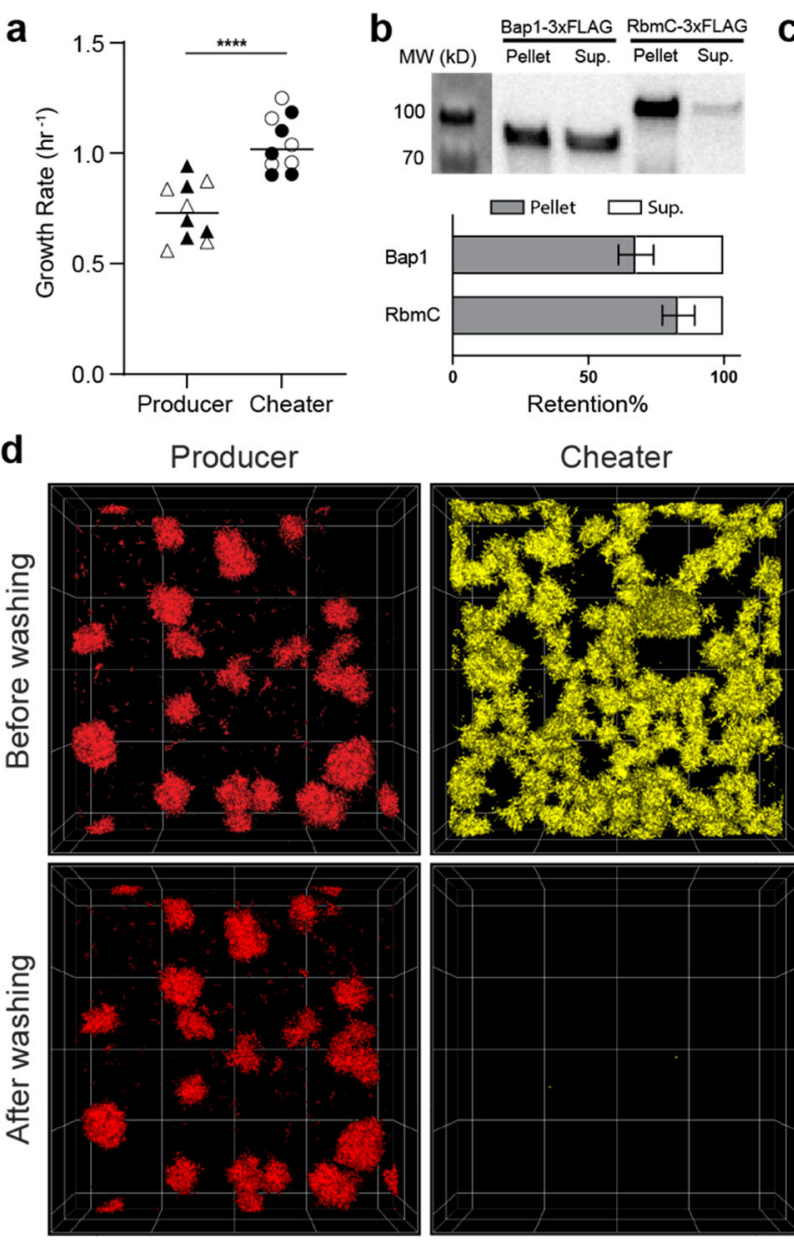

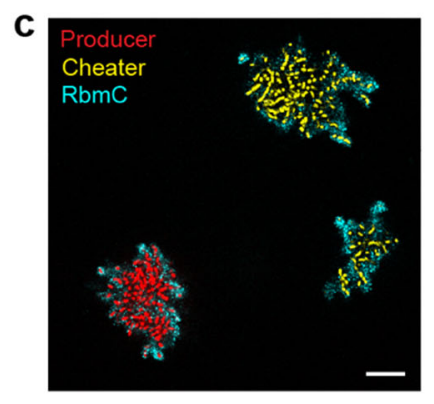

Producer + Cheater
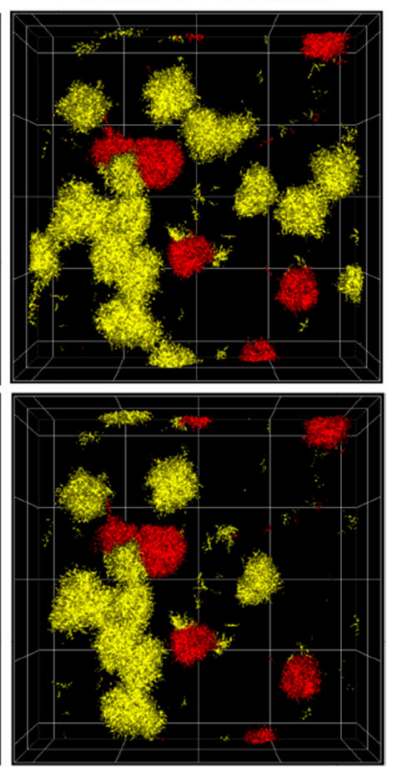

Fig. 1 RbmC and Bap1 are exploitable public goods in $V$. cholerae biofilms. a, Growth rates of the producer strain and the cheater strain $(\triangle \operatorname{bap} 1 \Delta \mathrm{rbmC})$ from $\mathrm{N}=10$ independent replicates $(* * * * \mathrm{P}<0.0001$, Mann-Whitney). Strains expressing mScarlet-I and mNeonGreen are shown by filled and empty markers, respectively. b, Top: Western blot showing Bap1 and RbmC in the biofilm (Pellet) and in the cell-free supernatant (Sup.). Bottom: quantification of the fraction of protein retained in biofilm $(\mathrm{N}=3)$. c, A representative confocal image $7 \mu \mathrm{m}$ above the substratum of a co-culture of producer (red) expressing $3 \times$ FLAG-tagged RbmC and cheater (yellow), stained with Cy3-conjugated anti-FLAG antibodies (cyan) to show the spatial distribution of RbmC. Scale bar: $10 \mu \mathrm{m}$. d, Mono-culture of producer (red), mono-culture of cheater (yellow), and co-culture of producer and cheater before and after washing. The producer constitutively expresses $\mathrm{mScartlet}-\mathrm{I}$ and the cheater constitutively expresses mNeonGreen. Image size: 222 


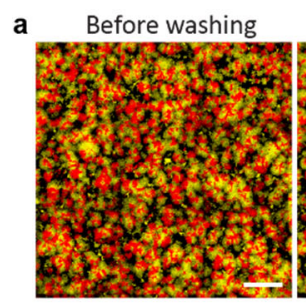

After washing
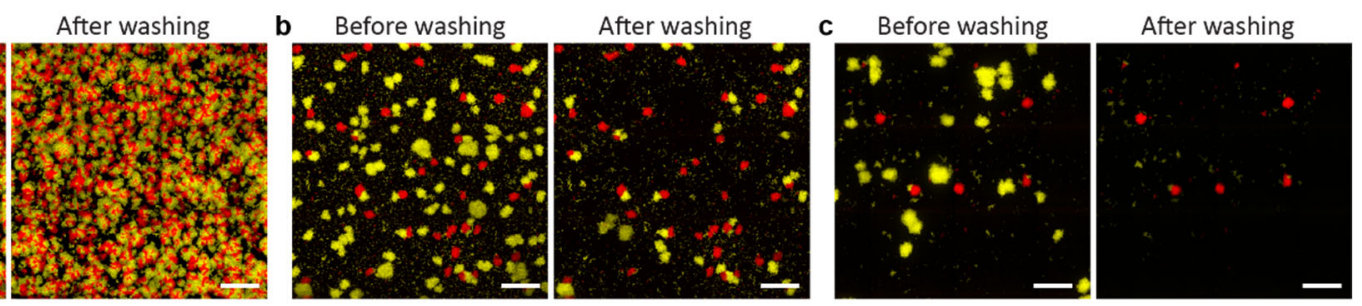

d
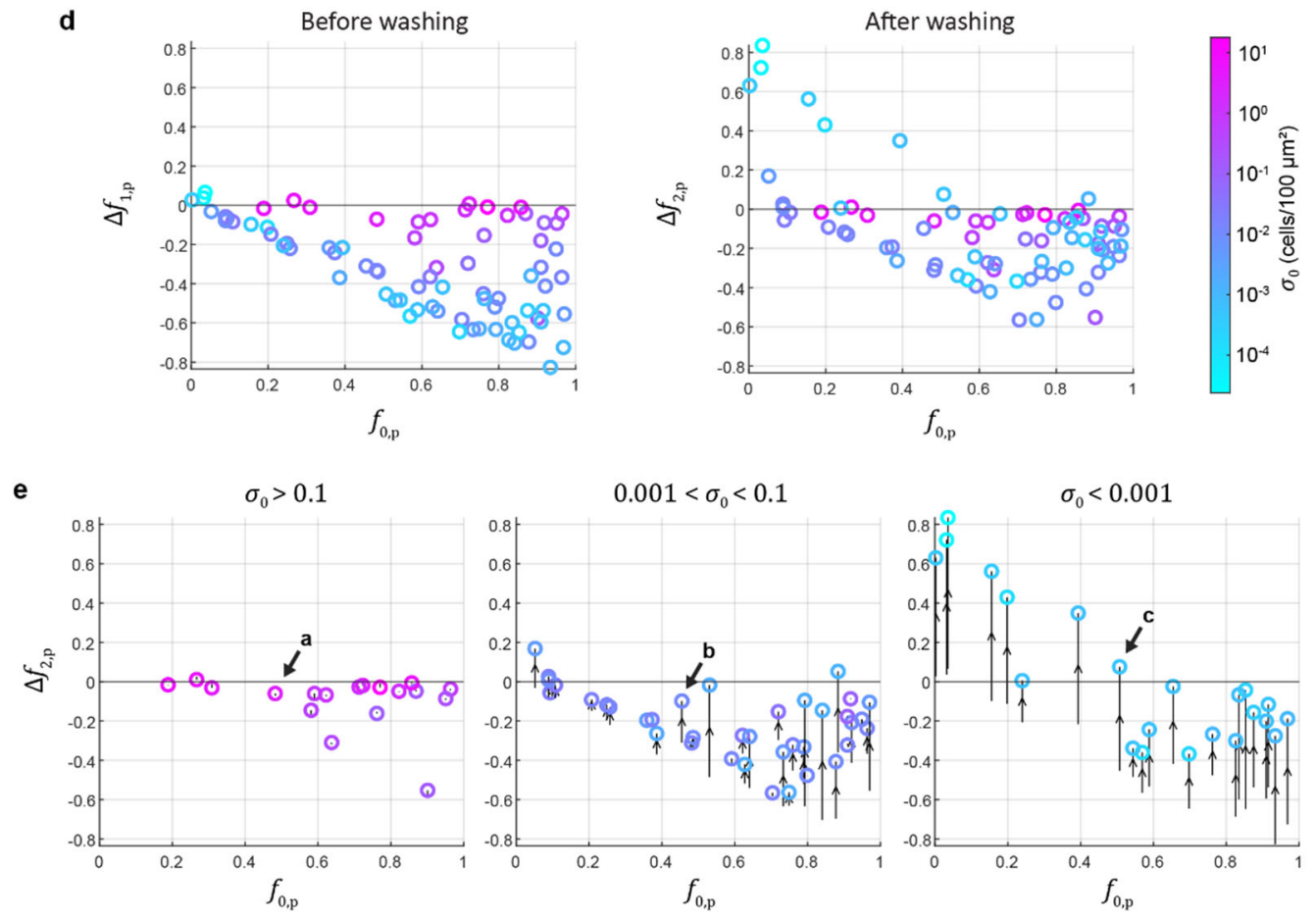

574 Fig. 2 | Competition between adhesion protein producer and cheater strains in a static environment. a-c, 575 Producer (red) and cheater (yellow) biofilms grown in a static environment before and after washing with 576 different inoculation number densities $\sigma_{0}$ corresponding to data labeled in (e). Scale bars: $100 \mu \mathrm{m}$. d, 577 Adhesion protein producers in competition with cheaters before and after washing. e, Adhesion protein 578 producers in competition with cheaters in different $\sigma_{0}$ ranges (color-coded) in a static environment. Each 579 data marker denotes the frequency change in producer after washing $\left(\Delta f_{2, \mathrm{p}}\right)$, and the corresponding frequency change before washing $\left(\Delta f_{1, \mathrm{p}}\right)$ is connected to it by an arrow $\left(\Delta f_{1, \mathrm{p}} \rightarrow \Delta f_{2, \mathrm{p}}\right)$. 
a

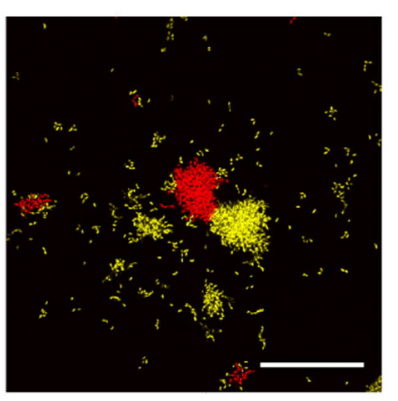

d

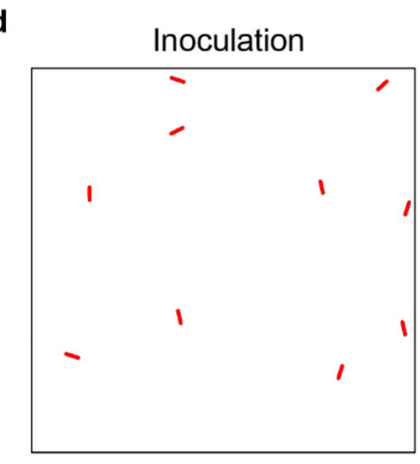

b
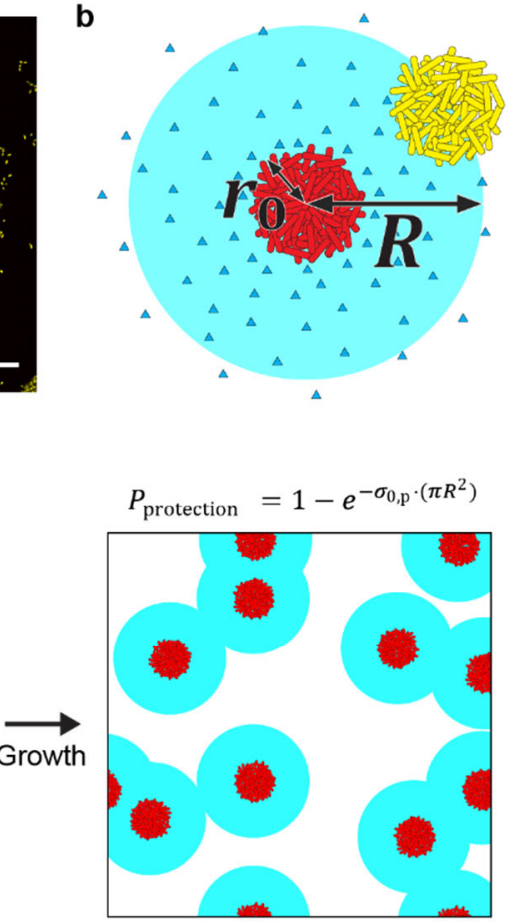

c

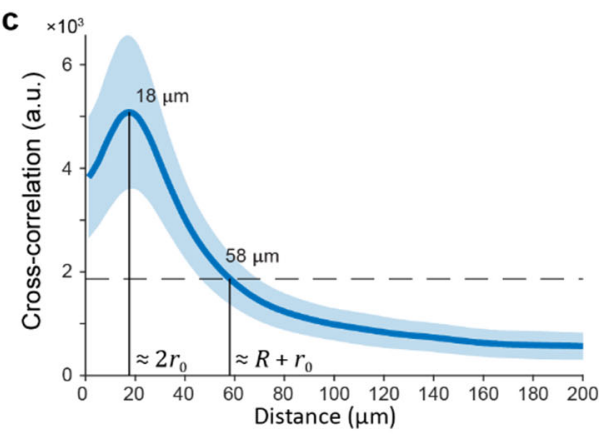

e

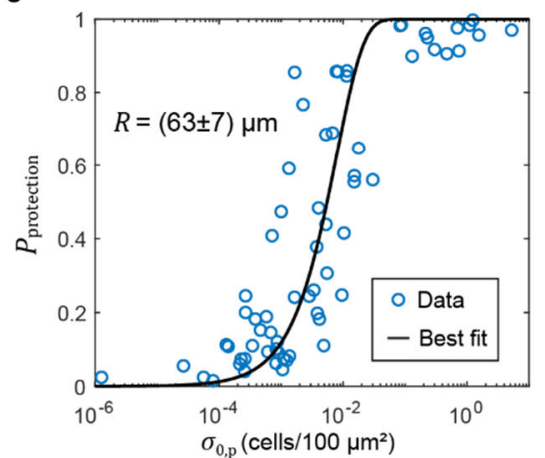

584

585

586

587

588

589

590

591

592

593

594

595

596

597

598

Fig. 3 | A spatial exploitation model quantitively explains competition dynamics. a, Representative confocal image of a co-culture of producer (red) and cheater (yellow). The maximum intensity projection image shows the distribution of cheater biomass around isolated producer clusters after washing. Scale bar: 50 $\mu \mathrm{m}$. b, Schematic of exploitation radius $R$ provided by diffusion of adhesion proteins from a producer cluster. $r_{0}$ is the physical radius of the producer cluster. c, Exploitation radius determined as $R=49 \pm 11$ $\mu \mathrm{m}$ by analyzing the cross-correlation between the distribution of producer and cheater biomasses. Error represents the standard error from $\mathrm{N}=4$ independent replicates. The peak at $18 \mu \mathrm{m}$ corresponds to $2 r_{0}$. The horizontal dashed line indicating $e^{-1}$ of the maximum cross-correlation intersects at $58 \mu \mathrm{m}$, corresponding to $R+r_{0}$. d, Schematic of the spatial model quantifying the protection probability $P_{\text {protection }}$ conferred by randomly distributed producer clusters. Each inoculated individual cell contributes to an area of protection around it and all producer clusters collectively yield $P_{\text {protection }}=1-\exp \left(-\sigma_{0, \mathrm{p}} \cdot \pi R^{2}\right)$, where $\sigma_{0, \mathrm{p}}$ is the inoculation number density of the producer. e, Experimentally measured $P_{\text {protection }}$ plotted vs. $\sigma_{0, \mathrm{p}}$. Fitting the data with the equation in (d) yields $R=63 \pm 7 \mu \mathrm{m}$ with $95 \%$ confidence bounds. 


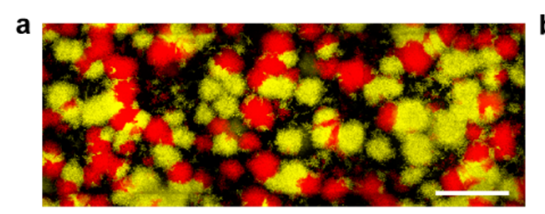

d

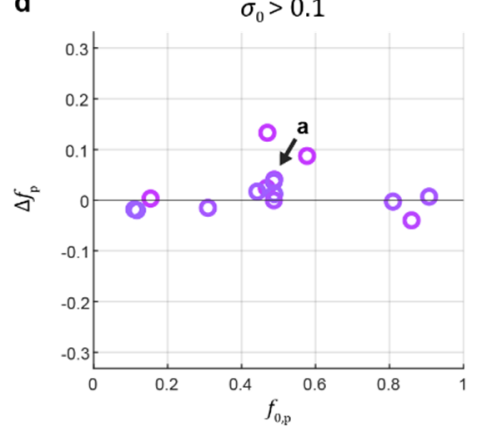

e

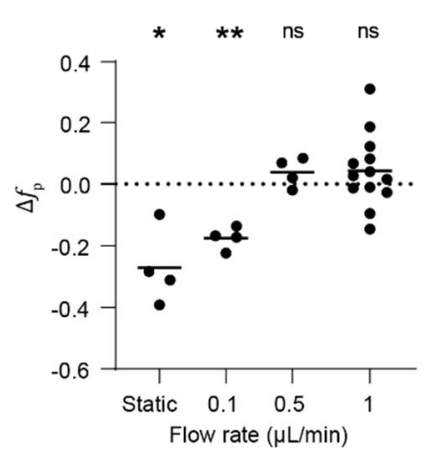

f

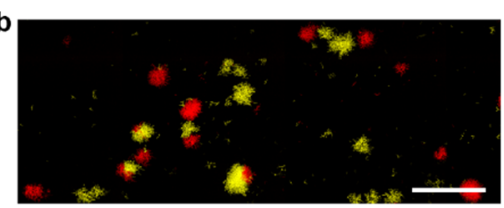

$0.01<\sigma_{0}<0.1$
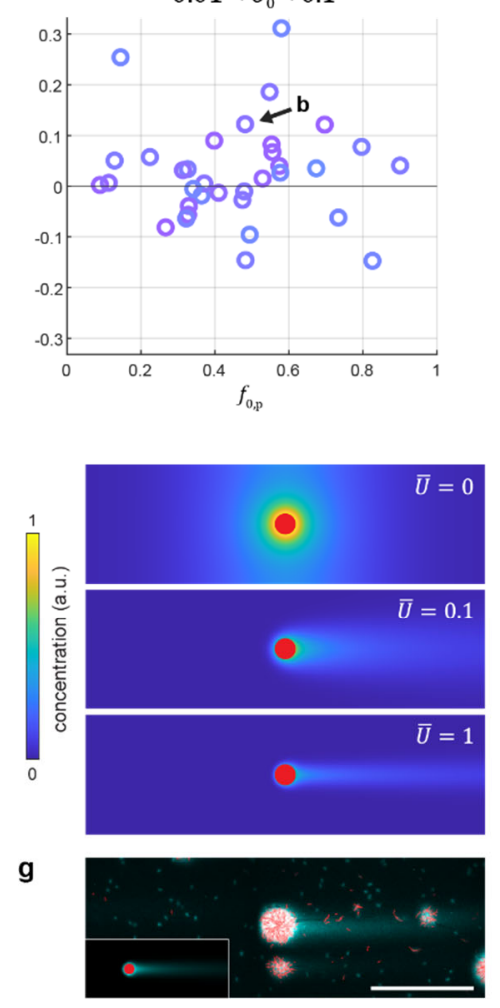

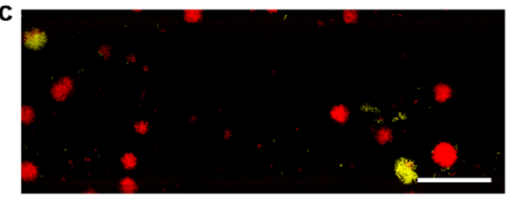

$\sigma_{0}<0.01$

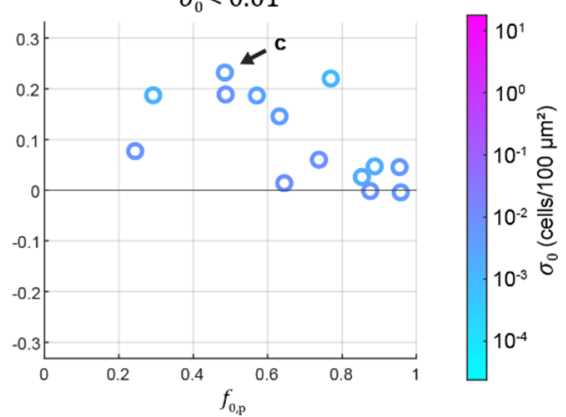

$\mathbf{h}$

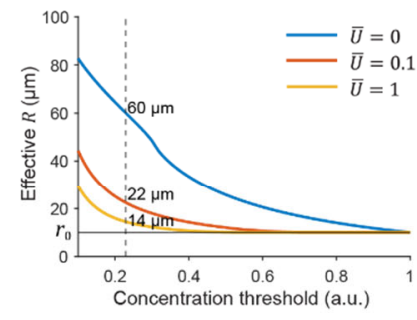

i

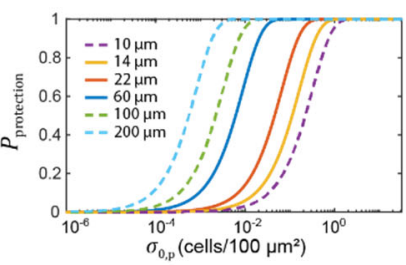

Fig. 4 Flow shifts the competition between producer and cheater by modulating the exploitation range of the adhesion protein. a-c, Representative maximum intensity projection images of producer (red) and cheater (yellow) biofilms grown in a microfluidic channel with different $\sigma_{0}$ corresponding to data labeled in (d). Scale bars: $100 \mu \mathrm{m}$. d, Producers in competition with cheaters at different $\sigma_{0}$ in a flow environment (flow rate $=1 \mu \mathrm{L} / \mathrm{min}$ ). e, Dependence of $\Delta f_{\mathrm{p}}$ on flow rate. Data labeled static were obtained from the static competition assay in 96-well plate. Data were taken at $0.01<\sigma_{0}<0.1$ and $0.4<f_{0, \mathrm{p}}<0.6$. One sample $t$-test was used to examine if $\Delta f_{\mathrm{p}}$ is statistically different from $0(* \mathrm{P}<0.05$ and $* * \mathrm{P}<0.01 ; \mathrm{N}=4,4,4$, and 13). f, Simulated depth-averaged adhesion protein concentration in a flow chamber at different average flow speeds $\bar{U}$. The concentration is normalized by the value at the edge of the producer cluster at $\bar{U}=0$. The red disk corresponds to a producer cluster of radius $r_{0}=10 \mu \mathrm{m}$. g, Distribution of adhesion protein Bap1 (cyan) around producer clusters (red) at the surface of the flow chamber shown by Cy3-conjugated anti-FLAG antibody staining. The corresponding simulation result is shown in the inset (Lower left). Scale bar: $100 \mu \mathrm{m}$. $\mathbf{h}$, Effective $R$ as a function of concentration threshold for protection at different flow rates in simulations. The horizontal line indicates the edge of the producer cluster at $r_{0}$. A threshold concentration of 0.23 (vertical dashed line) yields $R=60,22$, and $14 \mu \mathrm{m}$ for $\bar{U}=0,0.1$, and 1 , respectively. i, $P_{\text {protection }}$ vs. $\sigma_{0, \mathrm{p}}$ calculated for different $R$ values. 
619 1. Flemming, H.-C. et al. Biofilms: an emergent form of bacterial life. Nat. Rev. Microbiol. 14, 563575 (2016). $135-138(2001)$.

3. Persat, A. et al. The mechanical world of bacteria. Cell 161, 988-997 (2015). governs invasion resistance in bacterial biofilms. ISME J. 9, 1700-1709 (2015). transforms the landscape and community assembly of biofilms. Curr. Biol. 31, 2643-2651.e3 (2021). architecture confers individual and collective mechanisms of viral protection. Nat. Microbiol. 3, 26-31 (2018).

7. Hall-Stoodley, L., Costerton, J. W. \& Stoodley, P. Bacterial biofilms: from the natural environment to infectious diseases. Nat. Rev. Microbiol. 2, 95-108 (2004).

8. Barrasso, K. et al. Impact of a human gut microbe on Vibrio cholerae host colonization through biofilm enhancement. Preprint at https://www.biorxiv.org/content/10.1101/2021.02.01.429194v2 (2021). (2014). in Vibrio cholerae. Infect. Immun. 78, 3560-3569 (2010). biofilms adhere to surfaces. Front. Microbiol. 12, 1642 (2021). 
644

645

646

647

648

649

650

651

652

653

654

655

656

657

658

659

660

661

662

663

664

665

666

667

668

12. Nadell, C. D., Drescher, K. \& Foster, K. R. Spatial structure, cooperation, and competition in bacterial biofilms. Nat. Rev. Microbiol. 14, 589-600 (2016).

13. Cordero, O. X. \& Datta, M. S. Microbial interactions and community assembly at microscales. Curr. Opin. Microbiol. 31, 227-234 (2016).

14. Datta, M. S., Sliwerska, E., Gore, J., Polz, M. F. \& Cordero, O. X. Microbial interactions lead to rapid micro-scale successions on model marine particles. Nat. Commun. 7, 11965 (2016).

15. Ebrahimi, A., Schwartzman, J. \& Cordero, O. X. Cooperation and spatial self-organization determine rate and efficiency of particulate organic matter degradation in marine bacteria. Proc. Natl. Acad. Sci. USA 116, 23309-23316 (2019).

16. Stocker, R. \& Seymour, J. R. Ecology and physics of bacterial chemotaxis in the ocean. Microbiol. Mol. Biol. Rev. 76, 21 (2012).

17. West, S. A., Cooper, G. A., Ghoul, M. B. \& Griffin, A. S. Ten recent insights for our understanding of cooperation. Nat. Ecol. Evol. 5, 419-430 (2021).

18. Hamilton, W. D. The genetical evolution of social behaviour. I. J. Theor. Biol. 7, 1-16 (1964).

19. Hardin, G. The Tragedy of the Commons. Science 162, 1243-1248 (1968).

20. West, S. A., Diggle, S. P., Buckling, A., Gardner, A. \& Griffin, A. S. The social lives of microbes. Annu. Rev. Ecol. Evol. Syst. 38, 53-77 (2007).

21. Dal Co, A., van Vliet, S., Kiviet, D. J., Schlegel, S. \& Ackermann, M. Short-range interactions govern the dynamics and functions of microbial communities. Nat. Ecol. Evol. 4, 366-375 (2020).

22. Vliet, S. van, Hauert, C., Ackermann, M. \& Co, A. D. Global dynamics of microbial communities emerge from local interaction rules. Preprint at https://www.biorxiv.org/content/10.1101/2020.07.25.220822v2 (2021).

23. Julou, T. et al. Cell-cell contacts confine public goods diffusion inside Pseudomonas aeruginosa clonal microcolonies. Proc. Natl. Acad. Sci. USA 110, 12577-12582 (2013). 

(2013).

671

25. Nadell, C. D., Foster, K. R. \& Xavier, J. B. Emergence of spatial structure in cell groups and the evolution of cooperation. PLoS Comput. Biol. 6, e1000716 (2010).

26. Nadell, C. D. et al. Cutting through the complexity of cell collectives. Proc. R. Soc. B. 280, 20122770 (2013).

27. Drescher, K., Nadell, C. D., Stone, H. A., Wingreen, N. S. \& Bassler, B. L. Solutions to the public goods dilemma in bacterial biofilms. Curr. Biol. 24, 50-55 (2014).

28. Driscoll, W. W. \& Pepper, J. W. Theory for the evolution of diffusible external goods. Evolution 64, 2682-2687 (2010).

29. Allison, S. D. Cheaters, diffusion and nutrients constrain decomposition by microbial enzymes in spatially structured environments. Ecol. Lett. 8, 626-635 (2005).

30. Hamilton, W. D. The genetical evolution of social behaviour I \& II. J. Theor. Biol. 7, 1-52 (1964).

31. Rainey, P. B. \& Rainey, K. Evolution of cooperation and conflict in experimental bacterial populations. Nature 425, 72-74 (2003).

32. van Gestel, J., Weissing, F. J., Kuipers, O. P. \& Kovacs, A. T. Density of founder cells affects spatial pattern formation and cooperation in Bacillus subtilis biofilms. ISME J. 8, 2069-2079 (2014).

33. Irie, Y. et al. The Pseudomonas aeruginosa PSL polysaccharide is a social but noncheatable trait in biofilms. $m$ Bio 8, e00374-17 (2017).

34. Otto, S. B. et al. Privatization of biofilm matrix in structurally heterogeneous biofilms. mSystems 5, e00425-20 (2020).

35. Dragoš, A. et al. Collapse of genetic division of labour and evolution of autonomy in pellicle biofilms. Nat. Microbiol. 3, 1451-1460 (2018). 
36. Rossy, T., Nadell, C. D. \& Persat, A. Cellular advective-diffusion drives the emergence of bacterial surface colonization patterns and heterogeneity. Nat. Commun. 10, 2471 (2019).

37. Berk, V. et al. Molecular architecture and assembly principles of Vibrio cholerae biofilms. Science 337, 236-239 (2012).

38. Fong, J. C. N., Syed, K. A., Klose, K. E. \& Yildiz, F. H. Role of Vibrio polysaccharide (vps) genes in VPS production, biofilm formation and Vibrio cholerae pathogenesis. Microbiology 156, $2757-2769$ (2010).

39. Fong, J. C. et al. Structural dynamics of RbmA governs plasticity of Vibrio cholerae biofilms. eLife 6, (2017).

40. Nadell, C. D. \& Bassler, B. L. A fitness trade-off between local competition and dispersal in Vibrio cholerae biofilms. Proc. Natl. Acad. Sci. USA 108, 14181-14185 (2011). structure interact to determine spatial competition in Pseudomonas aeruginosa biofilms. eLife $\mathbf{6}$, (2017).

42. Madsen, J. S. et al. Facultative control of matrix production optimizes competitive fitness in Pseudomonas aeruginosa PA14 biofilm models. Appl. Environ. Microbiol. 81, 8414-8426

710 (2015).

43. Absalon, C., Van Dellen, K. \& Watnick, P. I. A communal bacterial adhesin anchors biofilm and bystander cells to surfaces. PLoS Pathog. 7, e1002210 (2011).

44. Beyhan, S. \& Yildiz, F. H. Smooth to rugose phase variation in Vibrio cholerae can be mediated by a single nucleotide change that targets c-di-GMP signalling pathway. Mol. Microbiol. 63, 9951007 (2007).

45. Yan, J., Sharo, A. G., Stone, H. A., Wingreen, N. S. \& Bassler, B. L. Vibrio cholerae biofilm growth program and architecture revealed by single-cell live imaging. Proc. Natl. Acad. Sci. USA 113, E5337-E5343 (2016). 
46. Alcolombri, U. et al. Sinking enhances the degradation of organic particles by marine bacteria. Nat. Geosci. 14, 775-780 (2021).

47. Diercks, A.-R. \& Asper, V. L. In situ settling speeds of marine snow aggregates below the mixed layer: Black Sea and Gulf of Mexico. Deep Sea Res. Part I Oceanogr. Res. Pap. 44, 385-398 (1997).

48. Nowald, N. et al. In-situ sinking speed measurements of marine snow aggregates acquired with a settling chamber mounted to the Cherokee ROV. in OCEANS 2009-EUROPE IEEE 1-6 (2009).

49. Drescher, K. et al. Architectural transitions in Vibrio cholerae biofilms at single-cell resolution. Proc. Natl. Acad. Sci. USA 113, E2066-E2072 (2016).

50. Hartmann, R. et al. Emergence of three-dimensional order and structure in growing biofilms. Nat. Phys. 15, 251-256 (2019). architectures. Preprint at https://www.biorxiv.org/content/10.1101/2021.08.06.455416v1 (2021). Sci. USA 118, e2107107118 (2021).

54. Nijjer, J. et al. Mechanical forces drive a reorientation cascade leading to biofilm self-patterning. Nat. Commun. 12, 6632 (2021). biofilm hyperinfectivity. Proc. Natl. Acad. Sci. USA 117, 11010-11017 (2020).

56. Kulthong, K. et al. Transcriptome comparisons of in vitro intestinal epithelia grown under static and microfluidic gut-on-chip conditions with in vivo human epithelia. Sci. Rep. 11, 3234 (2021).

57. Kirchberger, P. C. et al. Culture-independent tracking of Vibrio cholerae lineages reveals complex spatiotemporal dynamics in a natural population. Environ. Microbiol. 22, 4244-4256 (2020). 
59. Gardner, A. The Price equation. Curr. Biol. 18, R198-R202 (2008).

60. Pepper, J. W. Relatedness in trait croup models of social evolution. J. Theor. Biol. 206, 355-368 (2000).

61. Damore, J. A. \& Gore, J. Understanding microbial cooperation. J. Theor. Biol. 299, 31-41 (2012).

62. Driscoll, W. W. \& Pepper, J. W. Theory for the evolution of diffusible external goods. Evolution 64, 2682-2687 (2010).

63. Queller, D. C. Genetic relatedness in viscous populations. Evol. Ecol. 8, 70-73 (1994).

64. Brockhurst, M. A., Habets, M. G. J. L., Libberton, B., Buckling, A. \& Gardner, A. Ecological drivers of the evolution of public-goods cooperation in bacteria. Ecology 91, 334-340 (2010).

65. Rumbaugh, K. P. \& Sauer, K. Biofilm dispersion. Nat. Rev. Microbiol. 18, 571-586 (2020).

66. Callow, J. A. \& Callow, M. E. Trends in the development of environmentally friendly foulingresistant marine coatings. Nat. Commun. 2, 244 (2011).

67. Koschwanez, J. H., Foster, K. R. \& Murray, A. W. Sucrose utilization in budding yeast as a model for the origin of undifferentiated multicellularity. PLoS Biol. 9, e1001122 (2011).

68. Mitri, S., Xavier, J. B. \& Foster, K. R. Social evolution in multispecies biofilms. Proc. Natl. Acad. Sci. USA 108, 10839-10846 (2011).

69. Estrela, S. \& Brown, S. P. Community interactions and spatial structure shape selection on antibiotic resistant lineages. PLoS Comput. Biol. 14, e1006179 (2018).

70. Van Dyken, J. D., Muller, M. J. I., Mack, K. M. L. \& Desai, M. M. Spatial population expansion promotes the evolution of cooperation in an experimental prisoner's dilemma. Curr. Biol. 23, 919-923 (2013).

71. Frost, I. et al. Cooperation, competition and antibiotic resistance in bacterial colonies. ISME J. 12, 1582-1593 (2018).

72. Sharma, A. \& Wood, K. B. Spatial segregation and cooperation in radially expanding microbial colonies under antibiotic stress. ISME J. 15, 3019-3033 (2021). 
771

772

773

774

775

776

777

778

779

780

781

782

783

784

785

786

787

788

789

790

791

792

793

794

795

796

73. Griffin, A. S., West, S. A. \& Buckling, A. Cooperation and competition in pathogenic bacteria. Nature 430, 1024-1027 (2004).

74. Rumbaugh, K. P. et al. Quorum sensing and the social evolution of bacterial virulence. Curr. Biol. 19, 341-345 (2009).

75. van Gestel, J. et al. Short-range quorum sensing controls horizontal gene transfer at micron scale in bacterial communities. Nat. Commun. 12, 2324 (2021).

76. Darch, S. E. et al. Spatial determinants of quorum signaling in a Pseudomonas aeruginosa infection model. Proc. Natl. Acad. Sci. USA 115, 4779-4784 (2018).

77. Dalia, A. B., McDonough, E. \& Camilli, A. Multiplex genome editing by natural transformation. Proc. Natl. Acad. Sci. USA 111, 8937-8942 (2014).

78. Skorupski, K. \& Taylor, R. K. Positive selection vectors for allelic exchange. Gene 169, 47-52 (1996).

79. Hall, P. Introduction to the theory of coverage processes. (Wiley, 1988).

80. Fischer, P. F., Lottes, J. W. \& Kerkemeier, S. G. nek5000-web-page. NEK5000 Web page https://nek5000.mcs.anl.gov/ (2008).

81. Fischer, P. F. An Overlapping Schwarz method for spectral element solution of the incompressible Navier-Stokes equations. J. of Comput. Phys. 133, 84-101 (1997).

82. Mukherjee, S. \& Paul, M. R. Velocity and geometry of propagating fronts in complex convective flow fields. Phys. Rev. E 99, 012213 (2019).

83. Mukherjee, S. Front propagation and feedback in convective flow fields. (Virginia Polytechnic Institute and State University, 2020).

84. Paul, M. R., Chiam, K.-H., Cross, M. C., Fischer, P. F. \& Greenside, H. S. Pattern formation and dynamics in Rayleigh-Bénard convection: numerical simulations of experimentally realistic geometries. Physica D 184, 114-126 (2003). 